\title{
Imaging in Neurocritical Care Practice
}

\author{
Craig Williamson, MD, MS ${ }^{1}$ Larry Morgan, $\mathrm{DO}^{2} \quad$ Joshua P. Klein, MD, PhD 3
}

1 Department of Neurosurgery and Neurology, University of Michigan, Ann Arbor, Michigan

2 Department of Neurosurgery, University of Michigan,

Ann Arbor, Michigan

${ }^{3}$ Division of Hospital Neurology, Department of Neurology,

Brigham and Women's Hospital, Boston, Massachusetts

\begin{abstract}
Address for correspondence Joshua P. Klein, MD, PhD, Department of Neurology, Brigham and Women's Hospital, Room BB-334, 75 Francis Street, Boston, MA 02115 (e-mail: jpklein@bwh.harvard.edu).
\end{abstract}

Semin Respir Crit Care Med 2017;38:840-852.

\begin{abstract}
Keywords

- neuroimaging

- critical care

- ICU

The use of neuroimaging in conjunction with serial neurological examinations is a core component of modern neurocritical care practice. Although there is a growing role for other neuromonitoring techniques, the ability to quickly and accurately interpret images in the context of a patient's clinical status arguably remains the indispensable skill for neurocritical care practitioners. Due to its rapid acquisition time and excellent ability to detect intracerebral hemorrhage (ICH), cerebral edema, and signs of elevated intracranial pressure, computed tomography (CT) remains the most useful neuroimaging technique for intensive care unit (ICU) patients. An emergent head CT is obtained to inform most time-sensitive decisions that arise in the neurological ICU (NICU). CT features also figure prominently in prognostic scores for common NICU conditions such as traumatic brain injury (TBI), ICH, and subarachnoid hemorrhage (SAH). Among patients who are sufficiently stable to leave the ICU and lie flat for an extended period, magnetic resonance imaging provides much more detailed, high-contrast images which can aid in the detection of ischemia, diffuse axonal injury, and neuroprognostication. Though primarily used in neurocritical care research, nuclear medicine imaging techniques have some clinical applications, particularly in ancillary testing for brain death. Finally, as in the field of critical care as a whole, formal and point-of-care ultrasound studies are increasingly utilized in the NICU, and are an important tool in the neurointensivist's armamentarium. We review here the common applications of imaging in the neurocritical care setting. As ICU patients are frequently unstable and their risk of clinical decompensation increases substantially during transport away from the ICU, guidelines and recommendations for maximizing patient safety during transport to radiology studies are also explored.
\end{abstract}

\section{Computed Tomography and Structural Magnetic Resonance Imaging in Neurocritical Care}

Computed tomography (CT) and magnetic resonance imaging (MRI) are the most widely used neuroimaging modalities in general and in the neurological intensive care unit (NICU). A complete review of all of the many applications of
CT and MRI in NICU patients would be beyond the scope of this review; therefore, in the following section, a clinicoradiographic approach using CT and MRI for the most common neurocritical care conditions is presented. In general, in comparison to patients with neurological diseases in the outpatient or general inpatient setting, CT is obtained much more frequently than MRI in the NICU. As the clinical condition of NICU patients often evolves quickly, thereby
Issue Theme Advancements in Neurocritical Care and Emergency Neurology; Guest Editors: David Y. Hwang, MD, FNCS, and David M. Greer, MD, MA, FCCM, FAHA, FNCS, FAAN, FANA
Copyright (c) 2017 by Thieme Medical Publishers, Inc., 333 Seventh Avenue, New York, NY 10001, USA. Tel: +1(212) 584-4662. 
necessitating rapid evaluation and assessment prior to proceeding with medical or neurosurgical interventions, the major advantage of CT over MRI is the speed with which images are obtained. At many centers, portable head CT can be performed at the bedside, reducing risks associated with transport off of the unit and prolonged time lying flat.

Although CT is obtained more frequently, MRI has superior resolution and contrast between normal and abnormal brain; therefore, it can be extremely useful in many conditions. In particular, cytotoxic edema due to cerebral ischemia evolves slowly and is usually undetectable acutely on CT images, whereas acute ischemic changes are easily detected using MR diffusion-weighted sequences. Unfortunately, to obtain an MRI, patients must be laid flat in a narrow tube with a strong magnetic field for a minimum of several minutes, and access to support devices, such as endotracheal tubes, intravenous catheters (IVs), and bedside monitors is limited while the patient is in the scanner. Therefore, careful thought with regard to the anticipated benefit of MR data on patient care versus the risk of obtaining the scan should occur before any critically ill patient is transported to the MRI scanner.

\section{Imaging of Traumatic Brain Injury and Intracranial Hypertension}

CT is the imaging modality of choice for the initial evaluation of traumatic brain injury (TBI), and a head CT is typically obtained rapidly in the emergency room after screening and stabilization. Injuries commonly detected on CT include skull and facial fractures, epidural hematoma (EDH), subdural hematoma (SDH), subarachnoid hemorrhage (SAH), and cerebral contusions $^{1}$ (-Fig. 1). Localized and diffuse cerebral edema is also quite common. ${ }^{2}$ In contrast to spontaneous intracerebral hemorrhages (ICHs), there are frequently multiple locations and types of ICH in severe TBI. Trauma is the most common etiology for SAH. In contrast to aneurysmal etiologies, traumatic SAH tends to be thin and located at the cerebral convexities. When substantial subarachnoid blood is present in the basal cisterns, it is important to consider the possibility of aneurysm rupture leading to subsequent head trauma and to obtain appropriate angiographic imaging. ${ }^{3}$

The presence of cerebral contusions is a hallmark of TBI. As with contusions in other tissues, cerebral contusions are due to damaged, leaking small blood vessels. Contusions frequently start as multiple, small, scattered microhemorrhages with significant surrounding vasogenic edema. Red blood cells and plasma can continue to leak for a significant amount of time after the initial injury, leading to a characteristic delayed hematoma expansion that is often referred to as "blossoming" of the contusion. Due to this tendency to expand, close clinical monitoring and follow-up imaging are indicated in all patients with cerebral contusions. ${ }^{4-6}$ Typically, contusions are located frontally or in the anterior temporal lobes due to the tendency of the free-floating brain to scrape against bony prominences in the anterior and middle cranial fossae during trauma. Again, due to the free-floating nature of the brain within the skull, so-called coup injuries may be seen directly underlying the site of impact, with "contrecoup" contusions located 180 degrees from the impact. ${ }^{7}$

The current critical care management of patients with TBI is focused on avoiding secondary injuries, primarily by avoiding elevated intracranial pressure (ICP) and ensuring adequate cerebral perfusion pressure (CPP). Most studies of the treatment and monitoring of intracranial hypertension have been performed in patients with TBI. At present, accurate determination of ICP is dependent on invasive monitors, with CT data often used to guide decision making about monitor placement. In the 2007 Brain Trauma Foundation guidelines, it was recommended that all patients with severe TBI, defined as Glasgow's coma scale 3 to 8 , and an abnormal head CT receive ICP monitoring. An abnormal head CT was defined as one that reveals hematomas, contusions, swelling, herniation, or compressed basal cisterns. ${ }^{8}$ Since the publication of those guidelines, a randomized controlled trial in several medical centers in Bolivia and Ecuador failed to show that introduction of invasive ICP monitoring improved care in comparison to treatment guided by imaging and clinical examination. In the imaging

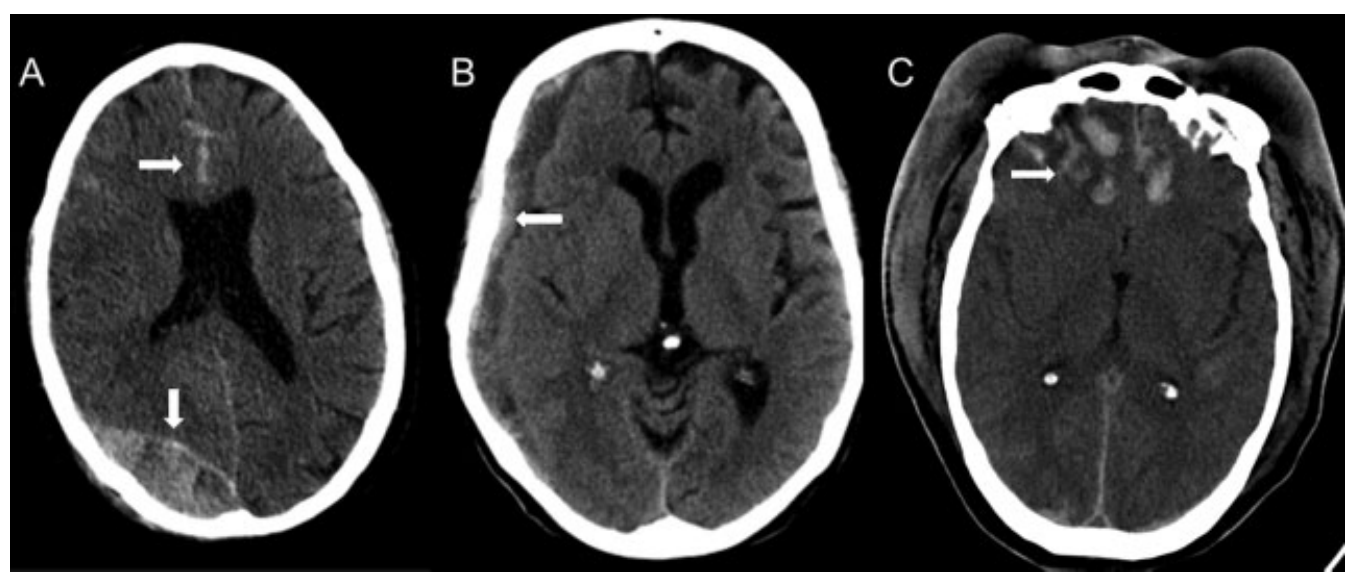

Fig. 1 Computed tomography appearance of traumatic brain injuries. (A) The vertical arrow indicates the location of a typical biconcave epidural hematoma, while the horizontal arrow indicates a contrecoup injury consisting of a small area of subarachnoid blood within the interhemispheric fissure; (B) a typical mixed density, holohemispheric acute-on-chronic subdural hematoma (arrow); and (C) the irregular, bifrontal hyperdensities with surrounding vasogenic edema are consistent with cerebral contusions (arrow). 
and clinical examination group, hyperosmolar therapy was administered if any of the following features suggestive of elevated ICP were present on CT: compressed perimesencephalic cisterns, midline shift, or cortical sulcal compression. Barbiturates were administered if the perimesencephalic cisterns remained compressed following hyperventilation and the administration of hyperosmolar therapy. ${ }^{9}$ Although this trial remains controversial and the generalizability of its results to North American medical centers is uncertain, it does suggest using CT features of elevated ICP to guide management if feasible.

While specific $\mathrm{CT}$ imaging features increase the likelihood of elevated ICP in TBI, no individual or set of features has proven highly sensitive and specific for intracranial hypertension. In an early study of patients in the NIH Traumatic Coma Databank, brain swelling with midline shift and compressed perimesencephalic cisterns were strongly correlated with elevated ICP. ${ }^{10}$ In another study, incremental worsening of sulcal effacement, subfalcine herniation, compressed basilar cisterns, and loss of gray-white differentiation were all linearly associated with elevations in ICP. There was also an association between progressively more slit-like ventricles and elevated ICP. ${ }^{11}$ This illustrates an important difference in imaging between TBI and various causes of hydrocephalus, in which elevated ICP is associated with progressive enlargement of the ventricles. Some authors have attempted to develop logistic regression models that use multiple $\mathrm{CT}$ features to predict elevated ICP. ${ }^{12}$ Recently, machine-learning methods utilizing multiple quantitative features derived from automated CT imaging analysis have been used to predict elevated ICP, but none of these methods has yet been validated and adapted into routine clinical practice. ${ }^{13,14}$

Several CT grading scales have been proposed, and CT imaging features are also a component of the most widely used prognostic models in TBI. The Marshall et al's CT classification ( - Table $\mathbf{1}$ ) was proposed in 1991 as a way of identifying patients at greatest risk of developing intracranial hypertension.

Table 1 The Marshall et al's CT classification for traumatic brain injury

\begin{tabular}{|c|c|}
\hline Category & Definition \\
\hline Diffuse injury I & $\begin{array}{l}\text { No visible intracranial pathology seen } \\
\text { on CT scan }\end{array}$ \\
\hline Diffuse injury II & $\begin{array}{l}\text { Cisterns are present with } \leq 5 \mathrm{~mm} \\
\text { midline shift; no high or mixed-density } \\
\text { lesions }>25 \mathrm{~mL}\end{array}$ \\
\hline Diffuse injury III & $\begin{array}{l}\text { Cisterns compressed with } \leq 5 \mathrm{~mm} \\
\text { midline shift; no high or mixed-density } \\
\text { lesions }>25 \mathrm{~mL}\end{array}$ \\
\hline Diffuse injury IV & $\begin{array}{l}\text { Midline shift }>5 \mathrm{~mm} \text {; no high or } \\
\text { mixed-density lesions }>25 \mathrm{~mL}\end{array}$ \\
\hline $\begin{array}{l}\text { Evacuated mass } \\
\text { lesion }\end{array}$ & Any lesion surgically evacuated \\
\hline $\begin{array}{l}\text { Nonevacuated } \\
\text { mass lesion }\end{array}$ & $\begin{array}{l}\text { High or mixed-density lesions }>25 \mathrm{~mL} \\
\text { not surgically evacuated }\end{array}$ \\
\hline
\end{tabular}

Abbreviation: CT, computed tomography.

Source: Adapted from Marshall et al. ${ }^{15}$
Table 2 The Rotterdam CT score for traumatic brain injury

\begin{tabular}{|l|l|}
\hline Predictor & \multicolumn{1}{l|}{ Score } \\
\hline Basal cisterns & 0 \\
\hline Normal & 1 \\
\hline Compressed & 2 \\
\hline Absent & 0 \\
\hline Midline shift & 1 \\
\hline None or $\leq 5 \mathrm{~mm}$ & \multicolumn{2}{|l|}{} \\
\hline$>5$ mm & 0 \\
\hline Epidural mass lesion & 1 \\
\hline Present & \multicolumn{1}{|l|}{} \\
\hline Absent & 0 \\
\hline IVH or traumatic SAH & 1 \\
\hline Absent & +1 \\
\hline Present &
\end{tabular}

Abbreviations: $\mathrm{CT}$, computed tomography; IVH, intraventricular hemorrhage; SAH, subarachnoid hemorrhage.

Source: Adapted from Maas et al. ${ }^{16}$

aThe sum score is added in order for scoring to correspond to the Marshall's CT classification.

It includes four classes of diffuse injury, which is defined as all patients except those with any high or mixed density lesions $>25 \mathrm{~mL}$. The four classes of diffuse injury are based on the presence or absence of compressed basal cisterns and midline shift. Separate categories are included for patients who have a mass lesion $>25 \mathrm{~mL}$, and those who have undergone surgical evacuation of a mass lesion. ${ }^{15}$ The Rotterdam score (- Table $\mathbf{2}$ ), first proposed in 2005, demonstrated that performance of the Marshall et al's score for mortality prediction could be improved by rearranging the individual $\mathrm{CT}$ characteristics, including more detailed differentiation of cisternal compression, and assigning points for the presence of EDH and intraventricular blood/ traumatic SAH. One point is added to the total to maintain the 1 to 6 categorization of the original Marshall et al's score. ${ }^{16}$

In contrast to other common TBIs, which are typically readily identifiable on $\mathrm{CT}$, diffuse axonal injury (DAI) is frequently occult on CT and is much more easily visualized with MRI. DAI is caused by the widespread shearing of axons due primarily to rotational forces. DAI is most commonly located in subcortical white matter at the gray-white junction, particularly in the frontal and temporal lobes. The corpus callosum is the second most commonly affected region followed by the brain stem, where white matter tracts in the dorsolateral midbrain and upper pons are most commonly injured. When present, DAI often occurs in multiple regions and may be extremely widespread. Macroscopic bleeding is present in approximately $20 \%$ of lesions, and these may be visible on CT as petechial hemorrhages within white matter tracts. ${ }^{17,18}$

In contrast to CT, MRI can detect nonhemorrhagic DAI and is therefore a much more sensitive test. Consequently, MRI should be considered in all stable patients with a severe and 
persistent impairment of consciousness. DAI can be visualized as areas of abnormal $\mathrm{T} 2$ prolongation on T2-weighted or fluid-attenuated inversion recovery (FLAIR) images. Diffusion-weighted images (DWI) can be used to detect acute areas of nonhemorrhagic DAI that sometimes are not detectable on T2/FLAIR images alone. ${ }^{19}$ As a small amount of bleeding is frequently present, imaging sequences with a high sensitivity for the detection of hemosiderin and other iron-containing hemoglobin breakdown products, such as gradient echo (GRE) and susceptibility-weighted (SW) sequences, are also useful to detect the presence of DAI in both the acute and chronic phases. ${ }^{20}$ In one series, $23 \%$ of DAI lesions were detected as a focal hypointensity on GRE did not have any corresponding DWI or T2/FLAIR changes. ${ }^{19}$ Using all of these MRI modalities improves the detection of DAI, but MRI remains an imperfect tool for detecting all areas of microscopic axonal injury. Diffuse tensor imaging works in a similar fashion to DWI by detecting areas where the diffusion of water molecules along axons is restricted, and has been used extensively as a research tool to identify DAI and correlate it with neurological outcome. ${ }^{21-23}$

\section{Imaging of Aneurysmal Subarachnoid Hemorrhage}

CT is the initial imaging modality of choice in all patients presenting with suspected SAH due to ruptured intracranial aneurysm, which classically presents following a "thunderclap headache," the abrupt onset of the worst headache of one's life, frequently associated with photophobia, nausea/ vomiting, nuchal rigidity, and altered mentation. When performed in the first 6 hours after symptom onset, the current generation of CT scanners were found to have a sensitivity of $98.7 \%$ (95\% confidence interval: $97.1-99.4 \%$ ) for detection of SAH, in a recent large systematic review. ${ }^{24}$ The sensitivity has been reported to approach $100 \%$ in the first 5 days in other case series, but is thought to substantially decrease at later timeframes. ${ }^{25}$ As the sensitivity of CT decreases over time and occasional early false negatives have been reported, current guidelines recommend performing lumbar punctures to evaluate for xanthochromia when clinical suspicion remains after a negative head $\mathrm{CT}^{26}{ }^{26}$ Some authors have reported improved detection of small SAHs with MRI, particularly in patients with a delayed presentation. Consequently, depending on the degree of clinical suspicion, MRI remains an option prior to pursuing LP, but false negatives also occur. ${ }^{27-29}$

Trauma remains the leading cause of SAH, but traumatic SAH is typically readily identifiable by a predominantly convexity hematoma in the setting of known trauma. In contrast, aneurysmal SAH is much more likely to present with diffuse subarachnoid blood with a substantial component in the basal cisterns and sometimes in the ventricles (-Fig. 2). Patients with SAH frequently lose consciousness at the time of ictus and may strike their head or, if they are driving, may experience a motor vehicle collision. Consequently, an aneurysmal etiology should always be considered when a patient presents with trauma and is found to have diffuse SAH with prominent cisternal involvement. Depending on the location of rupture and the direction of the jet of arterial blood, intraparenchymal

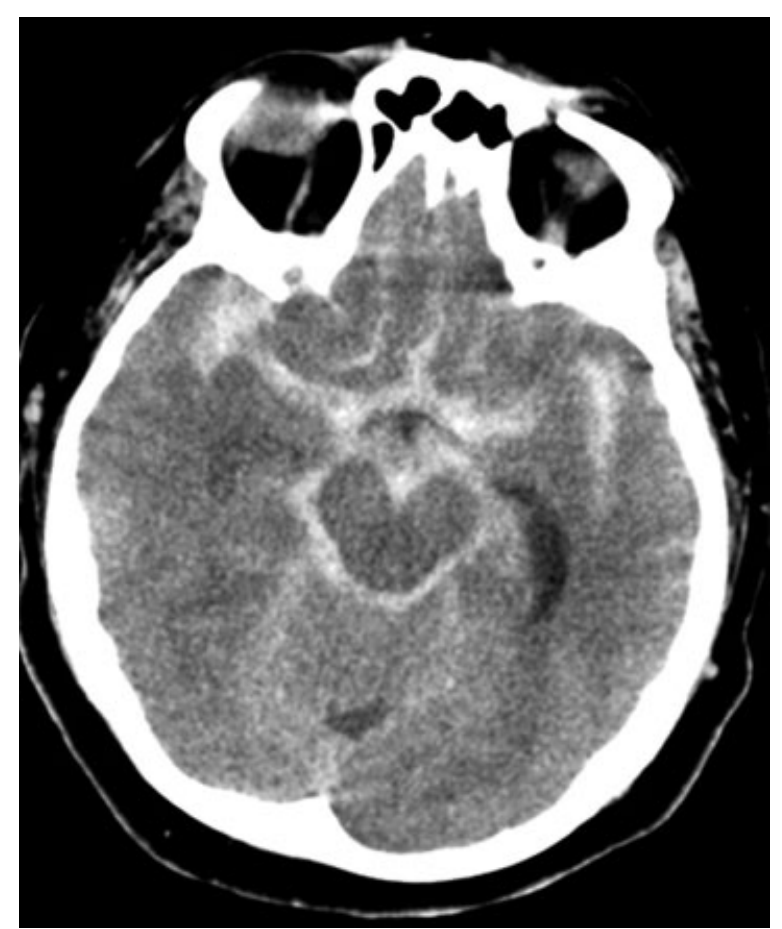

Fig. 2 A typical axial computed tomography appearance of aneurysmal subarachnoid hemorrhage, with thick clot layering in the basal cisterns, Sylvian fissure, and anterior interhemispheric fissure.

hemorrhage may occur and is often suggestive of aneurysm location. Classically, a ruptured aneurysm at the bifurcation of the middle cerebral artery (MCA) may present with a large temporal intraparenchymal hematoma with minimal blood in the Sylvian fissure. ${ }^{29}$ Intraventricular blood is present in nearly $20 \%$ of cases and SDH may also occur. ${ }^{30}$

In general, the spontaneous presence of cisternal blood is strongly suggestive of intracranial aneurysm rupture. However, a syndrome of nonaneurysmal perimesencephalic hemorrhage is well known and defined principally by its imaging features. In perimesencephalic SAH, blood is generally located in the cisterns anterior to the midbrain and pons, but can also be isolated to the quadrigeminal cistern. Blood may distribute into the third and fourth ventricles, but significant intraventricular or intraparenchymal hemorrhage should not be present. Although this distribution of hemorrhage can be due to a ruptured basilar artery aneur$\mathrm{ysm}$, an aneurysm is not present on angiography and patients generally have a benign course, without development of delayed cerebral ischemia (DCI). Rarely, obstructive hydrocephalus may occur necessitating ventriculostomy placement. The etiology of this syndrome is unknown, but given its relatively benign nature, a venous rupture is suspected. ${ }^{29}$

The amount of subarachnoid blood on initial imaging has long been recognized as a major risk factor for the development of DCI, and several CT grading scales have been developed to quantify this risk. In 1980, Fisher et al described a four-point scale based on subarachnoid hematoma thickness and the presence of intraparenchymal or intraventricular blood (-Table 3). Group 1 had no blood present on CT. Group 2 had thin, diffuse SAH without any vertical layers of hematoma 
Table 3 The Fisher's and modified Fisher's scales for predicting vasospasm in SAH

\begin{tabular}{|l|l|l|l|}
\hline \multicolumn{2}{|l|}{ Fisher's scale } & \multicolumn{2}{l|}{ Modified Fisher's scale } \\
\hline Grade & SAH appearance & Grade & Characteristics \\
\hline & & 0 & No SAH or IVH \\
\hline 1 & No SAH visible & 1 & Minimal/thin SAH, no IVH in both lateral ventricles \\
\hline 2 & Thin, diffuse SAH & 2 & Minimal/thin SAH, with IVH in both lateral ventricles \\
\hline 3 & Clot or thick SAH & & Thick SAH, no IVH in both lateral ventricles \\
\hline 4 & Diffuse or none, with IPH or IVH & 4 & Thick $^{\mathrm{b}}$ SAH, with IVH in both lateral ventricles \\
\hline
\end{tabular}

Abbreviations: IPH, intraparenchymal hemorrhage; IVH, intraventricular hemorrhage; $\mathrm{SAH}$, subarachnoid hemorrhage.

${ }^{a} G$ rade 3 is defined by localized clot and/or vertical layers of blood $\geq 1 \mathrm{~mm}$.

${ }^{\mathrm{b}}$ Thick $\mathrm{SAH}$ is defined as blood completely filling $\geq 1$ cistern or fissure.

more than $1 \mathrm{~mm}$ in thickness. Group 3 showed thick clots in the basal cisterns or hematoma of more than $1 \mathrm{~mm}$ vertical thickness, and had by far the highest incidence of clinical vasospasm. Group 4 consisted of the small number of patients who had either intraventricular or intraparenchymal hematoma without significant supratentorial subarachnoid blood. ${ }^{31}$

A modified Fisher's scale that addresses some of the deficiencies of the original scale was proposed in 2001 by Claassen et al $(-$ Table 3$)$. The initial Fisher's scale identified thick clot as a major risk factor for DCI, but did not include any additive risk of intraventricular or intraparenchymal hemorrhage, which were considered to constitute a separate class. The modified Fisher's scale ranges from 0 to 4 , with higher scores corresponding to greater DCI risk. Grade 0 patients have no SAH or IVH. Grade 1 has thin SAH and no IVH in both lateral ventricles, while grade 2 patients have thin SAH with IVH in both lateral ventricles. Grade 3 patients are those with thick SAH and no IVH in both lateral ventricles, while grade 4 patients have thick $\mathrm{SAH}$ with IVH in both lateral ventricles. Thick SAH is defined as hematoma filling $\geq 1$ cistern or fissure. ${ }^{32}$ An SAH sum score, proposed by Hijdra et al in 1990, was also found to strongly correlate with DCI. It is slightly more cumbersome to calculate than the Fisher's or modified Fisher's scores, but permits more precise quantification of the amount of subarachnoid blood. In this score, each of 10 basal cisterns and fissures as well as all four ventricles were assigned a score of 0 to 3 based on the quantity of blood in each one. The resulting score ranges from 0 to 42 and has been shown to be a better predictor of radiographic vasospasm than the modified Fisher's scale. ${ }^{33,34}$

\section{Imaging of Spontaneous Intracerebral Hemorrhages}

Patients with spontaneous (ICH) typically present with the acute onset of focal neurological deficits in a similar manner to ischemic stroke, though headache also occurs in slightly more than half patients with ICH. CT is the initial imaging modality of choice for all individuals presenting with the acute onset of neurological deficits, and it readily identifies acute $\mathrm{ICH}$ as a hyperdense mass lesion within the parenchyma. Approximately one-third of ICHs will significantly expand after initial presentation, mostly commonly within the first hour; therefore, a follow-up CT is typically performed..$^{35-37}$ The size and location of hemorrhage on initial CT, along with the clinical history, provide important clues about the underlying etiology.
Spontaneous ICH is considered to be either primary or secondary. Progressive vascular injury in response to hypertension is the most common cause of primary ICH and ICH in general. Cerebral amyloid angiopathy (CAA) is the other principal etiology of primary ICH. Compared with patients with CAA, hypertensive hemorrhages are more likely to be smaller, in a deep location, and occur in younger patients with a relevant past medical history. The caudate and putamen are the most common locations of hypertensive hemorrhages, followed by the thalamus, cerebellum, and pons. ${ }^{38}$ Hemorrhages due to CAA are more likely to be lobar and tend to occur more frequently in the occipital and parietal lobes.

Secondary causes of ICH are due to an underlying vascular malformation or structural lesion. Arteriovenous malformations (AVMs), cavernous malformations, dural AV fistulas, (dAVF), and aneurysms all can result in spontaneous ICH. Hemorrhages due to an underlying aneurysm usually have at least a small subarachnoid component, which should result in prompt investigations to rule out aneurysm, particularly in young patients with frontal or temporal hematomas. ${ }^{39}$ Hemorrhages due to underlying dAVF are more likely to include SAH in the cerebral convexity. In general, vascular imaging is indicated to rule out underlying vascular malformation in patients presenting with spontaneous ICH. Catheter angiography should be considered if an underlying small aneurysm or dural AVF is suspected. ${ }^{40}$

Underlying brain tumor is the other important cause of secondary ICH, which can occur in up to $10 \%$ of primary and metastatic tumors. Certain tumors have a greater propensity to bleed. Among primary brain tumors, glioblastoma and oligodendroglioma are associated with the greatest frequency of ICH. For metastatic tumors, melanoma, renal cell, thyroid, and choriocarcinomas are particularly likely to present with $\mathrm{ICH}^{39,41}$ Intratumoral hemorrhage can sometimes present with a blood/fluid level on $\mathrm{CT}$ or can be identified by the presence of hyperdense hematoma within or adjacent to a contrast-enhancing mass. Some authors have described an either hypodense or hyperdense tumor core visible within the clot, and the presence of significant early surrounding edema as important clues to underlying tumor on noncontrast $\mathrm{CT}^{42,43}$

Although CT is the imaging modality of choice for initial detection and acute follow-up for hematoma expansion, MRI is extremely useful for evaluating the underlying etiology and for 
detecting subacute or chronic hemorrhages. In particularly, the likelihood of CAA increases if small hemorrhages (microbleeds) are detected on sequences with high sensitivity for hemoglobin break-down products. As iron remains present in areas of prior hemorrhage, GRE and SW sequences have excellent sensitivity for detecting remote hemorrhages. According to Boston criteria, a diagnosis of probable CAA can be made when multiple hemorrhages in lobar, cortical, or corticosubcortical regions are detected on MRI in a patient older than 55 years. ${ }^{44}$ In general, contrast-enhanced MRI is preferred for detecting tumors. As a large ICH can obscure an underlying tumor, repeating an MRI in delayed fashion after hematoma resolution may permit tumor detection. Also, MRI is much more sensitive for detecting cavernous malformations, which frequently have a characteristic "popcorn" appearance on T2-weighted images, reflecting blood components in various stages of degradation, and are also readily visible on GRE and SW sequences. ${ }^{38}$

\section{Angiographic Imaging in the ICU}

Although conventional digital subtraction angiography (DSA) remains the gold standard for detecting cerebrovascular pathology, CT angiographic (CTA) and MR angiographic (MRA) images are increasingly superseding conventional angiography for many NICU conditions. It should be noted that conventional DSA is an invasive procedure that carries an approximately $2 \%$ risk of stroke or transient ischemic attack (TIA). ${ }^{45} \mathrm{CTA}$ has the advantage of rapid acquisition, but does expose patients to radiation and potentially nephrotoxic iodinated contrast. MRA with gadolinium contrast avoids radiation and is not nephrotoxic, though nephrogenic systemic fibrosis can rarely occur in patients with impaired renal function. Time-of-flight angiographic images can be obtained without gadolinium and provide good visualization of proximal arteries.

In the detection of ruptured aneurysms, one recent systematic review identified 50 studies where the sensitivity of CTA ranged from 86 to $100 \%$ in comparison with conventional angiography. The pooled sensitivity was $98 \%$ and specificity was $100 \%$. However, evidence of publication bias was also noted, which may have resulted in an overestimate of the sensitivity. ${ }^{46}$ In contrast, a more recent large single-center case series reported that the sensitivity of CTA in the setting of acute SAH was only $70.7 \%$ overall, and was $57.6 \%$ for aneurysms less than $5 \mathrm{~mm} .{ }^{47}$ Current SAH guidelines state that CTA may be considered as part of the initial work-up, as identification of an aneurysm on CTA will guide future treatment planning. However, if CTA is inconclusive, a DSA should be obtained. ${ }^{26}$ Because of its long acquisition time and low sensitivity for small aneurysms, MRA is generally not recommended for the acute evaluation of SAH. However, because it avoids radiation and iodinated contrast exposure, MRA is widely used as a screening tool in asymptomatic individuals. ${ }^{29}$

Less data are available regarding the sensitivity of detection for AVMs. However, one series found that the sensitivity for detecting ruptured AVMs was $87 \%$ for CTA, $83 \%$ for T2-weighted MRI, and 77\% for MRA in comparison to DSA. The sensitivities for unruptured AVMs were 96,97 , and $71 \%$, respectively. ${ }^{48}$ Based on the index of suspicion for an underlying vascular

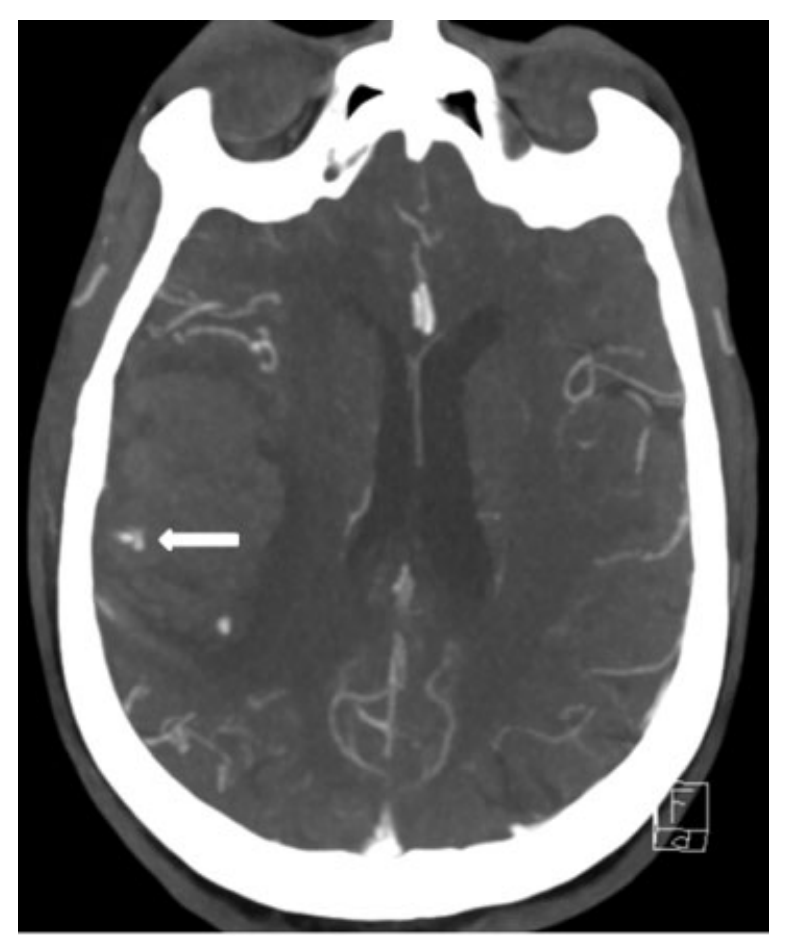

Fig. 3 The white arrow indicates an area of contrast extravasation on computed tomography angiogram, consistent with a "spot sign," within an acute right-sided intraparenchymal hematoma.

abnormality, a CTA is generally sufficient as a screening tool for patients presenting with spontaneous ICH. The presence of contrast extravasation within the hematoma on CT angiogram is termed the "spot sign," and is strongly associated with an increased probability of significant hematoma expansion and worsened neurological outcome $\mathrm{H}^{49}$ ( - Fig. 3). If SAH, abnormal vessels, or another reason to clinically suspect a vascular malformation is present, DSA should be considered. Prominent early vasogenic edema, location next to a dural sinus, hyperdensity within a venous sinus on noncontrast $\mathrm{CT}$, or absence of sinus opacification on contrasted CT should prompt venous imaging with MR or CT venogram.

Aside from the initial evaluation of hemorrhagic and ischemic stroke, angiographic imaging is also widely used to diagnose cerebral vasospasm in aneurysmal SAH. The relationship between angiographic vasospasm and $\mathrm{DCI}$ is complex and a detailed discussion is beyond the scope of this article. However, angiographic narrowing visualized on CTA or DSA occurs in up to $70 \%$ of patients with aneurysmal SAH, and has not consistently been correlated with poor outcome. Less than half of patients with radiographic vasospasm will develop clinical signs of DCI and, in up to $15 \%$ of cases, DCI can occur in the absence of radiographic vasospasm..$^{50,51}$ Nonetheless, new unexplained decline in the level of consciousness or focal neurological deficit is much more likely to be due to $\mathrm{DCl}$ when angiographic vasospasm is present. Initial screening with CTA is often employed, but if the clinical suspicion for DCI is high, some patients will immediately undergo catheter angiography. In this case, the presence of radiographic vasospasm can be diagnosed and immediately treated with the direct injection of vasodilatory agents, such 
as nicardipine or milrinone, into spasmed arteries. In extreme cases, balloon angioplasty may be employed to open up severely narrowed vessels. ${ }^{26,52}$

\section{Neurocritical Care Applications of Perfusion Imaging} Various radiological methods for assessing cerebral blood flow (CBF) have been widely used in neurocritical care research, and have been particularly useful in understanding the interaction between brain injury and cerebral autoregulation. Positron emission tomography (PET) and single-photon emission computed tomography (SPECT) were frequently used in earlier research studies, and PET has been trialed as a patient selection method for extracranial-intracranial bypass surgery. ${ }^{53}$ However, in NICU clinical practice, the use of nuclear medicine perfusion studies is primarily limited to the verification of brain death. Brain perfusion scintigraphy for the diagnosis of brain death typically utilizes technetium-99m. Although three-dimensional SPECT images may better visualize the cerebellum and brain stem, where thyroid and salivary gland perfusion may mimic intracranial blood flow, these typically require patient transport, which may be challenging for unstable patients. Twodimensional static images can be obtained rapidly at the patient bedside, and the finding of a "hollow" nonperfused skull is considered to be $100 \%$ specific for brain death. ${ }^{54}$

Beyond nuclear medicine perfusion studies, the advent of CT- and MR-based perfusion techniques have significantly expanded the role of perfusion imaging in clinical practice. CT perfusion requires slightly more radiation than conventional noncontrast CTs, but significantly less than PET or SPECT. ${ }^{55}$ Both $\mathrm{CT}$ and MR perfusion are increasingly being used for selecting acute stroke patients most likely to benefit from endovascular thrombolysis. ${ }^{56-58}$ However, in the ICU setting, perfusion scans are primarily used to evaluate for DCI in SAH. Given its rapid acquisition time, wide availability, and low cost, CT perfusion is the more commonly used modality, but both CT and MR perfusion are endorsed in recent guidelines. ${ }^{26}$

CT perfusion works by acquiring spiral CT images, while iodinated contrast passes through the cerebral vasculature. The propagation of density changes in individual voxels during the passage of contrast is used to quantify mean transit time (MTT), CBF, and cerebral blood volume (CBV) in regions of interest. The total CBV in a region of interest is equal to the CBF times the MTT. Regions with impaired perfusion due to cerebral vasospasm are expected to show decreased CBF and increased MTT, though absolute thresholds consistent with DCI have not been defined, and likely vary between scanners and institutions. ${ }^{55,59}$ Some small, single-center studies have suggested that CT perfusion is more sensitive and specific for diagnosing DCI than CTA alone. ${ }^{60}$ However, perfusion deficits occur more frequently in areas where severe vasospasm is present on CTA and the two tests are frequently performed together. ${ }^{61-63}$ Consequently, the exact role for $\mathrm{CT}$ perfusion in addition to CTA and DSA is unclear, and institutional protocols vary significantly.

\section{Functional MRI}

In contrast to structural MRI, functional MRI(fMRI) techniques are not widely used in neurocritical care practice, though they are frequently used as research tools in relevant disease processes. fMRI measures changes in blood oxygen level dependent signals to brain regions as a surrogate for changes in regional brain activity related to cognitive tasks, and can also be used to detect networks of highly connected brain regions. Recent work has found a correlation between functional connectivity in default mode networks and levels of consciousness. ${ }^{64}$ Another study identified several patients, previously thought to be in a persistent vegetative state, where fMRI was used to detect voluntary modulation of brain activity in response to instructions. ${ }^{65}$ In the future, it may be possible to use fMRI to assess and predict recovery of consciousness, but at present, such applications remain investigative.

\section{Ultrasound in Neurocritical Care}

Bedside ultrasound is increasingly being incorporated into intensive care practice. In neurocritical care, transcranial Doppler (TCD) ultrasonography has long been used for several cerebrovascular applications. In addition, bedside ultrasonography can rapidly provide neurointensivists with valuable information about cardiopulmonary sequelae of neurological disease.

\section{Transcranial Doppler Ultrasonography}

TCD ultrasound has a multitude of applications in critically ill patients including the detection of vasospasm, particularly in aneurysmal SAH, detection of stenosis or occlusion in acute stroke patients, detection of "right-to-left" intracardiac shunting, assessment of collateral flow pathways, and of vasomotor reactivity. ${ }^{66}$ TCD is very operator dependent, requiring the operator to adjust to varying anatomy and other circumstances that can interfere with accurate and usable data collection. Though the specific TCD equipment can vary, it typically consists of a 1 - to $5-\mathrm{MHz}$ transducer with M-mode and spectral Doppler capabilities.

TCD provides a noninvasive and relatively risk-free means of determining blood flow velocity through several intracranial and extracranial vessels, making it a useful tool in screening for vasospasm in aneurysmal SAH patients (-Fig. 4). ${ }^{67}$ Changes in vessel's lumen diameter are inversely proportional to the velocity of blood flow through the vessel, allowing for an inferred method to detect vasospasm. In comparison reviews utilizing conventional angiography, mean flow velocities of more than $120 \mathrm{~cm} / \mathrm{s}$ in the MCA correlated with significant vasospasm on angiography with a sensitivity of 77.2\%; likewise, average MCA velocities of more than $200 \mathrm{~cm} / \mathrm{s}$ correlated with more than $50 \%$ narrowing of the vessels lumen on angiography. ${ }^{67,68}$ The use of TCD has allowed physicians to predict which patients are at greatest risk for developing DCI. Of note, the majority of patients who develop vasospasm will not experience delayed ischemic symptoms, and, rarely, DCI can develop in the absence of large-vessel vasospasm. However, the development of new neurological deficits in conjunction with vasospasm on TCD increases the likelihood that DCI is present. Hyperdynamic states are common in aneurysmal SAH and an important confounder of velocities on TCD. To help eliminate this, the ratio of the MCA to extracranial ICA, also termed the 


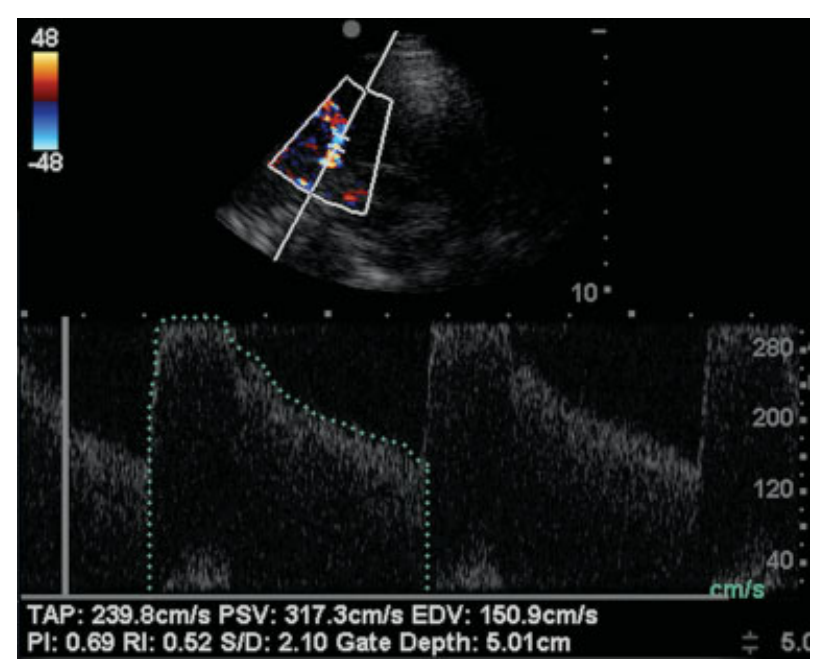

Fig. 4 Screenshot of acquisition of transcranial Doppler signals using bedside point-of-care ultrasound in a patient with subarachnoid hemorrhage and severe vasospasm. The upper panel shows acquisition of the Doppler signal from the left middle cerebral artery. The bottom panel shows a manual tracing of the arterial waveform with calculated values of average velocity, or time average peak (TAP), peak systolic velocity (PSV), end-diastolic velocity (EDV), pulsatility index (PI), resistive index (RI), and systolic/diastolic ratio (S/D).

"Lindegaard ratio" can be helpful. MCA/ICA mean velocity ratios of more than 3.0 have been reported to be highly sensitive for angiographic vasospasm. ${ }^{68}$

High-intensity transient signals (HITSs) are another useful feature of TCD. Thromboses, gaseous bubbles, and/or particulate matter larger than erythrocytes will reflect ultrasonic waves more intensely, which is the basis for HITS and its ability to identify real-time embolic events. These emboli can often be identified by sound, as the HITS frequently make a clicking or chirping sound. ${ }^{69}$ TCD has a similar sensitivity and specificity for detecting right-to-left intracardiac shunting as transesophageal echocardiography, and can be completed without the need for sedation or additional invasive maneuvers aside from injection of agitated saline from a peripheral IV. ${ }^{70,71}$ The combination of both techniques has been reported to be $100 \%$ sensitive for identifying both intraand extracardiac right-to-left shunts. ${ }^{72}$

The use of TCD ultrasonography in the verification of basilar artery flow in those patients with a clinically suspected basilar artery occlusion appears to be useful only for proximal occlusions. The overall sensitivity was $72 \%$ with a specificity of $94 \%$; however, the sensitivity is weak for distal occlusions, limiting its usefulness in the context of basilar artery syndromes and should not be relied upon as a single modality to exclude basilar artery occlusions. ${ }^{73,74}$

\section{Bedside Ultrasound Evaluation of Neurocardiogenic Injury}

Cardiomyopathies are a well-documented complication of severe neurologic injury, occurring in up to $17 \%$ of neurologically injured patients. Having a way to rapidly assess the level of injury and accurately assess ejection fraction can impact the management of neurologic injury, most notably in situations involving vasospasm management following aneurysmal SAH. The typical presentation of neurocardiogenic injury on bedside ultrasound involves hypokinesis from apical wall motion abnormalities that tend to spare the base. Approximately 65\% of patients will present in this fashion, though other regional wall motion abnormalities remain common. Another $26 \%$ of patients have right ventricular involvement. ${ }^{75}$ Diagnostic criteria for stress-induced cardiomyopathy, also known as Takotsubo's cardiomyopathy, have been proposed ( - Table 4). ${ }^{76}$

\section{Bedside Ultrasound for Pulmonary Edema}

Lung ultrasound (LUS) has proved to be a valuable tool for the bedside evaluation of multiple pulmonary-related conditions in critically ill patients. One of the most commonly encountered conditions in the ICU is alveolar pulmonary edema. LUS evaluation provides a rapid assessment based on the presence of "B-lines" or "comet-tail" artifacts, which are suggestive of alveolar pulmonary edema (-Fig. 5). ${ }^{77}$ Ultrasound changes suggestive of pulmonary edema can precede radiographic changes, and may be useful to guide fluid management for SAH patients receiving hemodynamic augmentation for cerebral vasospasm. ${ }^{78}$ Three or more B-lines is generally considered to be consistent with pulmonary edema. The presence of B-lines does not differentiate the type of pulmonary edema (cardiogenic vs. neurogenic vs. others). The mechanism for neurogenic pulmonary edema has not been well elucidated; however, there appears to be a strong association with the level of sympathetic activation and catecholamine release. The incidence is also unknown, but studies have suggested that somewhere between 2 and 43\% of acutely neurologically injured patients have some degree of neurogenic pulmonary edema. Some disorders, such as sudden unexplained death in epilepsy have between 80 and 100\% of individuals showing signs of neurogenic pulmonary edema at autopsy. ${ }^{79}$

Table 4 Proposed Mayo criteria for diagnosing stress cardiomyopathy

1. Transient hypokinesis, akinesis, or dyskinesis of the left ventricular mid-segments with or without apical involvement; the regional wall motion abnormalities extend beyond a single epicardial vascular distribution

2. Absence of obstructive coronary disease or angiographic evidence of acute plaque rupture

3. New electrocardiogram abnormalities (either ST-segment elevation and/or T-wave inversion) or modest elevation in troponin

4. Absence of pheochromocytoma and myocarditis

Source: Adapted from Prasad et al. ${ }^{76}$ 


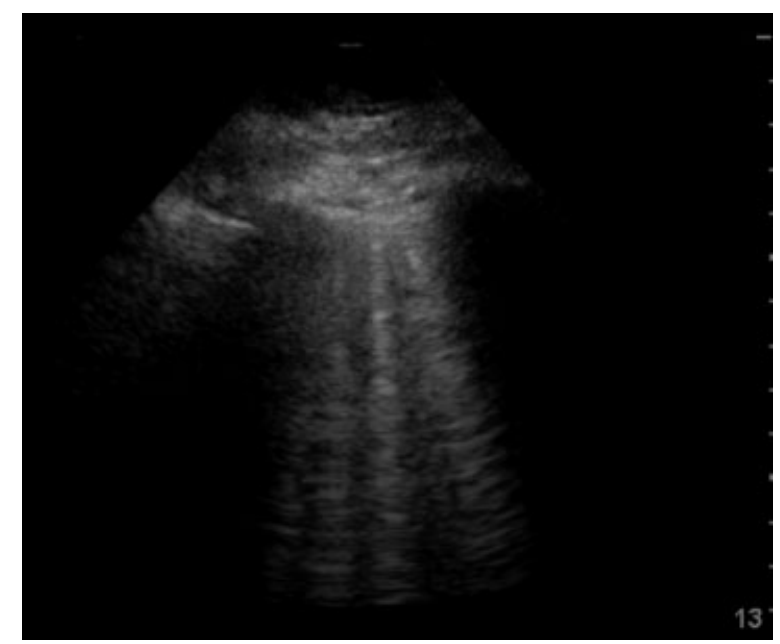

Fig. 5 Ultrasound image of the right lung in a patient with subarachnoid hemorrhage and pulmonary edema. The B-lines can be identified as linear, echogenic structures that start at the pleura (top of image) and extend vertically the length of the image.

\section{Safety Issues in Neurocritical Care Imaging}

Caution should be exercised when transporting any critically ill patient for imaging studies, as transport is independently associated with increased risk of adverse outcomes. ${ }^{80}$ Due to the risk of exacerbating underlying elevations in ICP during movement or when lying flat for extended periods, patients with brain injuries face additional transport-related risks. Sometimes neuroimaging data are necessary to make critical management decisions, in which case the transport of unstable patients may be justified. However, clinicians should always exercise independent judgment in determining when the benefits of obtaining imaging outweigh the risks of transfer.

Few formal guidelines exist regard the transport of patients with neurological injuries, but individual hospitals frequently develop their own transport protocols. The European Society of Intensive Care Medicine proposed recommendations for the intrahospital transport of patients with severe head injury in 1999, but updated guidelines have not been published since then. ${ }^{81}$ The Society of Critical Care Medicine published guidelines for the intrahospital transport of critically ill patients in $2004 .{ }^{82}$ Both of these guidelines highlight the need to ensure that patients are stabilized as much as possible prior to transport. For brain injured patients, this includes ensuring acceptable oxygenation, blood pressure, ICP, and CPP. A respiratory therapist should be present during the transport of intubated patients. Ideally, an ACLS certified physician with advanced airway skills should accompany any unstable patients undergoing emergent imaging. All monitoring devices used in the ICU environment should be continued and essential supplies, including basic resuscitation drugs, should be present. For patients at risk of intracranial hypertension, mannitol and/or hypertonic saline should be on hand. When transport ventilators are not available, bag mask ventilation is often employed. However, caution should be exercised in ensuring that hypoventilation resulting in elevated carbon dioxide and intracranial hypertension, as well as significant hyperventilation that results in decreased $\mathrm{CBF}$, does not occur. End-tidal $\mathrm{CO}_{2}$ monitoring may be helpful in this regard.

More recent publications have emphasized the importance of checklists in ensuring patient safety during transport. ${ }^{83}$ However, comprehensive checklists for neurocritical care patients have not been published. In many NICUs, portable head CTs are employed to decrease the risks of patient transport, and some single-center studies have suggested that these devices decrease the risk of complications and minimize physiological abnormalities. ${ }^{84,85}$ In the case of MRI scanning, neurocritical care physicians should carefully weigh the risks of patient transport with the immediate benefit of obtaining MRI data. MRI scans require patients to remain flat for significantly longer periods than CT, and the patient's airway is difficult to access while in the scanner. In addition, due to the strong magnetic field, some important monitoring devices, such as intraparenchymal ICP monitors, may result in significant artifact or may not be compatible. Consequently, MRI should be deferred in patients with unstable hemodynamics or oxygenation, significant risk of intracranial hypertension, or high risk of loss of airway protective reflexes.

\section{Conclusion}

The care provided for patients in the neurointensive care unit is comprehensive, and imaging remains an integral part of providing such care. In many instances, imaging allows for rapid and even at-the-bedside clinical decision making that can dramatically impact the outcome of patients. CT remains the most robust and utilized imaging modality in neurointensive care units due to its speed, the broad structural information it can provide, and relative safety profile. Structural MRI is also widely used, though its utility remains somewhat limited as its time and positioning requirements mandate careful selection of critically ill patients for which it would be a safe imaging modality. Functional imaging techniques such as those used in fMRI and nuclear medicine studies are primarily used in research, but can provide additional objective information for families in the determination of brain function/death. Formal and bedside ultrasonography has solidified itself in the critical care realm for its rapid assessment negating the need to place patients at risk during transport, avoidance of radiation, and cost-effectiveness. Its limitations remain in the experience of the operator/interpreter. Nevertheless, its utility is likely to increase in the years ahead as its useful applications continue to expand.

\section{References}

1 Bodanapally UK, Sours C, Zhuo J, Shanmuganathan K. Imaging of traumatic brain injury. Radiol Clin North Am 2015;53(04):695-715 , viii

2 Saatman KE, Duhaime AC, Bullock R, Maas AI, Valadka A, Manley GT; Workshop Scientific Team and Advisory Panel Members. Classification of traumatic brain injury for targeted therapies. J Neurotrauma 2008;25(07):719-738

3 Edlow JA, Caplan LR. Avoiding pitfalls in the diagnosis of subarachnoid hemorrhage. N Engl J Med 2000;342(01):29-36

4 Peterson EC, Chesnut RM. Talk and die revisited: bifrontal contusions and late deterioration. J Trauma 2011;71(06):1588-1592 
5 Alahmadi H, Vachhrajani S, Cusimano MD. The natural history of brain contusion: an analysis of radiological and clinical progression. J Neurosurg 2010;112(05):1139-1145

6 Kurland D, Hong C, Aarabi B, Gerzanich V, Simard JM. Hemorrhagic progression of a contusion after traumatic brain injury: a review. J Neurotrauma 2012;29(01):19-31

7 Young LA, Rule GT, Bocchieri RT, Burns JM. Biophysical mechanisms of traumatic brain injuries. Semin Neurol 2015;35(01):5-11

8 Brain Trauma Foundation; American Association of Neurological Surgeons and Congress of Neurological Surgeons. Guidelines for the management of severe traumatic brain injury. J Neurotrauma 2007;24(Suppl 1):S1-S106

9 Chesnut RM, Temkin N, Carney N, et al; Global Neurotrauma Research Group. A trial of intracranial-pressure monitoring in traumatic brain injury. N Engl J Med 2012;367(26):2471-2481

10 Eisenberg HM, Gary HE Jr, Aldrich EF, et al. Initial CT findings in 753 patients with severe head injury. A report from the NIH Traumatic Coma Data Bank. J Neurosurg 1990;73(05):688-698

11 Miller MT, Pasquale M, Kurek S, et al. Initial head computed tomographic scan characteristics have a linear relationship with initial intracranial pressure after trauma. J Trauma 2004;56(05): 967-972, discussion 972-973

12 Mizutani T, Manaka S, Tsutsumi H. Estimation of intracranial pressure using computed tomography scan findings in patients with severe head injury. Surg Neurol 1990;33(03):178-184

13 Chen W, Cockrell CH, Ward K, Najarian K. Predictability of intracranial pressure level in traumatic brain injury: features extraction, statistical analysis and machine learning-based evaluation. Int J Data Min Bioinform 2013;8(04):480-494

14 Chen W, Belle A, Cockrell C, Ward KR, Najarian K. Automated midline shift and intracranial pressure estimation based on brain CT images. J Vis Exp 2013;74(74):3871

15 Marshall LF, Marshall SB, Klauber MR, et al. A new classification of head injury based on computerized tomography. J Neurosurg 1991;75(01):S14-S20

16 Maas AI, Hukkelhoven CW, Marshall LF, Steyerberg EW. Prediction of outcome in traumatic brain injury with computed tomographic characteristics: a comparison between the computed tomographic classification and combinations of computed tomographic predictors. Neurosurgery 2005;57(06):1173-1182, discussion 1173-1182

17 Gentry LR. Imaging of closed head injury. Radiology 1994;191 (01):1-17

18 Gentry LR, Godersky JC, Thompson B. MR imaging of head trauma: review of the distribution and radiopathologic features of traumatic lesions. AJR Am J Roentgenol 1988;150(03):663-672

19 Huisman TA, Sorensen AG, Hergan K, Gonzalez RG, Schaefer PW. Diffusion-weighted imaging for the evaluation of diffuse axonal injury in closed head injury. J Comput Assist Tomogr 2003;27(01): 5-11

20 Parizel PM, Ozsarlak O, Van Goethem JW, et al. Imaging findings in diffuse axonal injury after closed head trauma. Eur Radiol 1998;8 (06):960-965

21 Wang JY, Bakhadirov K, Devous MD Sr, et al. Diffusion tensor tractography of traumatic diffuse axonal injury. Arch Neurol 2008;65(05):619-626

22 Huisman TA, Schwamm LH, Schaefer PW, et al. Diffusion tensor imaging as potential biomarker of white matter injury in diffuse axonal injury. AJNR Am J Neuroradiol 2004;25(03):370-376

23 Ware JB, Hart T, Whyte J, Rabinowitz A, Detre JA, Kim J. Intersubject variability of axonal injury in diffuse traumatic brain injury. J Neurotrauma 2017;34(14):2243-2253

24 Dubosh NM, Bellolio MF, Rabinstein AA, Edlow JA. Sensitivity of early brain computed tomography to exclude aneurysmal subarachnoid hemorrhage: a systematic review and meta-analysis. Stroke 2016;47(03):750-755

25 Cortnum S, Sørensen P, Jørgensen J. Determining the sensitivity of computed tomography scanning in early detection of subarachnoid hemorrhage. Neurosurgery 2010;66(05):900-902, discussion 903
26 Connolly ES Jr, Rabinstein AA, Carhuapoma JR, et al; American Heart Association Stroke Council; Council on Cardiovascular Radiology and Intervention; Council on Cardiovascular Nursing; Council on Cardiovascular Surgery and Anesthesia; Council on Clinical Cardiology. Guidelines for the management of aneurysmal subarachnoid hemorrhage: a guideline for healthcare professionals from the American Heart Association/American Stroke Association. Stroke 2012;43(06):1711-1737

27 Shimoda M, Hoshikawa K, Shiramizu H, Oda S, Matsumae M. Problems with diagnosis by fluid-attenuated inversion recovery magnetic resonance imaging in patients with acute aneurysmal subarachnoid hemorrhage. Neurol Med Chir (Tokyo) 2010;50 (07):530-537

28 Fiebach JB, Schellinger PD, Geletneky K, et al. MRI in acute subarachnoid haemorrhage; findings with a standardised stroke protocol. Neuroradiology 2004;46(01):44-48

29 van Gijn J, Kerr RS, Rinkel GJ. Subarachnoid haemorrhage. Lancet 2007;369(9558):306-318

30 Adams HP Jr, Kassell NF, Torner JC, Sahs AL. CT and clinical correlations in recent aneurysmal subarachnoid hemorrhage: a preliminary report of the Cooperative Aneurysm Study. Neurology 1983;33(08):981-988

31 Fisher CM, Kistler JP, Davis JM. Relation of cerebral vasospasm to subarachnoid hemorrhage visualized by computerized tomographic scanning. Neurosurgery 1980;6(01):1-9

32 Claassen J, Bernardini GL, Kreiter K, et al. Effect of cisternal and ventricular blood on risk of delayed cerebral ischemia after subarachnoid hemorrhage: the Fisher scale revisited. Stroke 2001;32(09):2012-2020

33 Hijdra A, Brouwers PJ, Vermeulen M, van Gijn J. Grading the amount of blood on computed tomograms after subarachnoid hemorrhage. Stroke 1990;21(08):1156-1161

34 Dupont SA, Wijdicks EF, Manno EM, Lanzino G, Rabinstein AA. Prediction of angiographic vasospasm after aneurysmal subarachnoid hemorrhage: value of the Hijdra sum scoring system. Neurocrit Care 2009;11(02):172-176

35 Brott T, Broderick J, Kothari R, et al. Early hemorrhage growth in patients with intracerebral hemorrhage. Stroke 1997;28(01):1-5

36 Dowlatshahi D, Demchuk AM, Flaherty ML, Ali M, Lyden PL, Smith EE; VISTA Collaboration. Defining hematoma expansion in intracerebral hemorrhage: relationship with patient outcomes. Neurology 2011;76(14):1238-1244

37 Hemphill JC III, Greenberg SM, Anderson CS, et al; American Heart Association Stroke Council; Council on Cardiovascular and Stroke Nursing; Council on Clinical Cardiology. Guidelines for the Management of Spontaneous Intracerebral Hemorrhage: A Guideline for Healthcare Professionals From the American Heart Association/ American Stroke Association. Stroke 2015;46(07):2032-2060

38 Smith EE, Rosand J, Greenberg SM. Hemorrhagic stroke. Neuroimaging Clin N Am 2005;15(02):259-272, ix

39 Fewell ME, Thompson BG, Hoff JT. Spontaneous intracerebral hemorrhage: a review. Neurosurg Focus 2003;15:1-16

40 Gandhi D, Chen J, Pearl M, Huang J, Gemmete JJ, Kathuria S. Intracranial dural arteriovenous fistulas: classification, imaging findings, and treatment. AJNR Am J Neuroradiol 2012;33(06):1007-1013

41 Kondziolka D, Bernstein M, Resch L, et al. Significance of hemorrhage into brain tumors: clinicopathological study. J Neurosurg 1987;67(06):852-857

42 Iwama T, Ohkuma A, Miwa Y, et al. Brain tumors manifesting as intracranial hemorrhage. Neurol Med Chir (Tokyo) 1992;32(03): 130-135

43 Little JR, Dial B, Bélanger G, Carpenter S. Brain hemorrhage from intracranial tumor. Stroke 1979;10(03):283-288

44 Knudsen KA, Rosand J, Karluk D, Greenberg SM. Clinical diagnosis of cerebral amyloid angiopathy: validation of the Boston criteria. Neurology 2001;56(04):537-539

45 Cloft HJ, Joseph GJ, Dion JE. Risk of cerebral angiography in patients with subarachnoid hemorrhage, cerebral aneurysm, 
and arteriovenous malformation: a meta-analysis. Stroke 1999; 30(02):317-320

46 Westerlaan HE, van Dijk JM, Jansen-van der Weide MC, et al. Intracranial aneurysms in patients with subarachnoid hemorrhage: CT angiography as a primary examination tool for diagnosis-systematic review and meta-analysis. Radiology 2011;258 (01):134-145

47 Philipp LR, McCracken DJ, McCracken CE, et al. Comparison Between CTA and Digital Subtraction Angiography in the Diagnosis of Ruptured Aneurysms. Neurosurgery 2017;80(05): 769-777

48 Gross BA, Frerichs KU, Du R. Sensitivity of CT angiography, T2-weighted MRI, and magnetic resonance angiography in detecting cerebral arteriovenous malformations and associated aneurysms. J Clin Neurosci 2012;19(08):1093-1095

49 Wada R, Aviv RI, Fox AJ, et al. CT angiography "spot sign" predicts hematoma expansion in acute intracerebral hemorrhage. Stroke 2007;38(04):1257-1262

50 Frontera JA, Fernandez A, Schmidt JM, et al. Defining vasospasm after subarachnoid hemorrhage: what is the most clinically relevant definition? Stroke 2009;40(06):1963-1968

51 Nolan CP, Macdonald RL. Can angiographic vasospasm be used as a surrogate marker in evaluating therapeutic interventions for cerebral vasospasm? Neurosurg Focus 2006;21(03):E1-E8

52 Greenberg ED, Gold R, Reichman M, et al. Diagnostic accuracy of CT angiography and CT perfusion for cerebral vasospasm: a metaanalysis. AJNR Am J Neuroradiol 2010;31(10):1853-1860

53 Powers WJ, Clarke WR, Grubb RL Jr, Videen TO, Adams HP Jr, Derdeyn CP; COSS Investigators. Extracranial-intracranial bypass surgery for stroke prevention in hemodynamic cerebral ischemia: the Carotid Occlusion Surgery Study randomized trial. JAMA 2011;306(18):1983-1992

54 Conrad GR, Sinha P. Scintigraphy as a confirmatory test of brain death. Semin Nucl Med 2003;33(04):312-323

55 Harrigan MR, Leonardo J, Gibbons KJ, Guterman LR, Hopkins LN. CT perfusion cerebral blood flow imaging in neurological critical care. Neurocrit Care 2005;2(03):352-366

56 Saver JL, Goyal M, Bonafe A, et al; SWIFT PRIME Investigators. Stent-retriever thrombectomy after intravenous t-PA vs. t-PA alone in stroke. N Engl J Med 2015;372(24):2285-2295

57 Campbell BC, Mitchell PJ, Kleinig TJ, et al; EXTEND-IA Investigators. Endovascular therapy for ischemic stroke with perfusionimaging selection. N Engl J Med 2015;372(11):1009-1018

58 Lansberg MG, Christensen S, Kemp S, et al; CT Perfusion to Predict Response to Recanalization in Ischemic Stroke Project (CRISP) Investigators. Computed Tomographic Perfusion to Predict Response to Recanalization in Ischemic Stroke. Ann Neurol 2017;81 (06):849-856

59 Sanelli PC, Ugorec I, Johnson CE, et al. Using quantitative CT perfusion for evaluation of delayed cerebral ischemia following aneurysmal subarachnoid hemorrhage. AJNR Am J Neuroradiol 2011;32(11):2047-2053

60 Dankbaar JW, de Rooij NK, Velthuis BK, Frijns CJ, Rinkel GJ, van der Schaaf IC. Diagnosing delayed cerebral ischemia with different CT modalities in patients with subarachnoid hemorrhage with clinical deterioration. Stroke 2009;40(11):3493-3498

61 Wintermark M, Ko NU, Smith WS, Liu S, Higashida RT, Dillon WP. Vasospasm after subarachnoid hemorrhage: utility of perfusion $\mathrm{CT}$ and $\mathrm{CT}$ angiography on diagnosis and management. AJNR Am J Neuroradiol 2006;27(01):26-34

62 Aralasmak A, Akyuz M, Ozkaynak C, Sindel T, Tuncer R. CT angiography and perfusion imaging in patients with subarachnoid hemorrhage: correlation of vasospasm to perfusion abnormality. Neuroradiology 2009;51(02):85-93

63 Sanelli PC, Pandya A, Segal AZ, et al. Cost-effectiveness of CT angiography and perfusion imaging for delayed cerebral ischemia and vasospasm in aneurysmal subarachnoid hemorrhage. AJNR Am J Neuroradiol 2014;35(09):1714-1720
64 Lee MH, Smyser CD, Shimony JS. Resting-state fMRI: a review of methods and clinical applications. AJNR Am J Neuroradiol 2013; 34(10):1866-1872

65 Monti MM, Vanhaudenhuyse A, Coleman MR, et al. Willful modulation of brain activity in disorders of consciousness. N Engl J Med 2010;362(07):579-589

66 American College of Radiology (ACR); Society for Pediatric Radiology (SPR); Society of Radiologists in Ultrasound (SRU). AIUM practice guideline for the performance of a transcranial Doppler ultrasound examination for adults and children. JUltrasound Med 2012;31(09):1489-1500

67 Marshall SA, Nyquist P, Ziai WC. The role of transcranial Doppler ultrasonography in the diagnosis and management of vasospasm after aneurysmal subarachnoid hemorrhage. Neurosurg Clin $\mathrm{N}$ Am 2010;21(02):291-303

68 Gonzalez NR, Boscardin WJ, Glenn T, Vinuela F, Martin NA Vasospasm probability index: a combination of transcranial Doppler velocities, cerebral blood flow, and clinical risk factors to predict cerebral vasospasm after aneurysmal subarachnoid hemorrhage. J Neurosurg 2007;107(06):1101-1112

69 Sliwka U, Job F-P, Wissuwa D, et al. Occurrence of transcranial Doppler high-intensity transient signals in patients with potential cardiac sources of embolism. A prospective study. Stroke 1995;26(11):2067-2070

70 Droste DW, Reisener M, Kemény V, et al. Contrast transcranial Doppler ultrasound in the detection of right-to-left shunts. Reproducibility, comparison of 2 agents, and distribution of microemboli. Stroke 1999;30(05):1014-1018

71 Nemec JJ, Marwick TH, Lorig RJ, et al. Comparison of transcranial Doppler ultrasound and transesophageal contrast echocardiography in the detection of interatrial right-to-left shunts. Am J Cardiol 1991;68(15):1498-1502

72 Komar M, Olszowska M, Przewłocki T, et al. Transcranial Doppler ultrasonography should it be the first choice for persistent foramen ovale screening? Cardiovasc Ultrasound 2014;12:16

73 Brandt T, Knauth M, Wildermuth S, et al. CT angiography and Doppler sonography for emergency assessment in acute basilar artery ischemia. Stroke 1999;30(03):606-612

74 Kermer P, Wellmer A, Crome O, Mohr A, Knauth M, Bähr M. Transcranial color-coded duplex sonography in suspected acute basilar artery occlusion. Ultrasound Med Biol 2006;32(03): 315-320

75 Roshanzamir S, Showkathali R. Takotsubo cardiomyopathy a short review. Curr Cardiol Rev 2013;9(03):191-196

76 Prasad A, Lerman A, Rihal CS. Apical ballooning syndrome (TakoTsubo or stress cardiomyopathy): a mimic of acute myocardial infarction. Am Heart J 2008;155(03):408-417

77 Lichtenstein D, Mézière G, Biderman P, Gepner A, Barré O. The comet-tail artifact. An ultrasound sign of alveolar-interstitial syndrome. Am J Respir Crit Care Med 1997;156(05):1640-1646

78 Williamson CA, Co I, Pandey AS, Gregory Thompson B, Rajajee V. Accuracy of daily lung ultrasound for the detection of pulmonary edema following subarachnoid hemorrhage. Neurocrit Care 2016; 24(02):189-196

79 Davison DL, Terek M, Chawla LS. Neurogenic pulmonary edema. Crit Care 2012;16(02):212

80 Schwebel C, Clec'h C, Magne S, et al; OUTCOMEREA Study Group. Safety of intrahospital transport in ventilated critically ill patients: a multicenter cohort study*. Crit Care Med 2013;41(08): 1919-1928

81 Ferdinande P; Working Group on Neurosurgical Intensive Care of the European Society of Intensive Care Medicine. Recommendations for intra-hospital transport of the severely head injured patient. Intensive Care Med 1999;25(12):1441-1443

82 Warren J, Fromm RE Jr, Orr RA, Rotello LC, Horst HM; American College of Critical Care Medicine. Guidelines for the inter- and intrahospital transport of critically ill patients. Crit Care Med 2004;32(01):256-262 
83 Brunsveld-Reinders AH, Arbous MS, Kuiper SG, de Jonge E. A comprehensive method to develop a checklist to increase safety of intra-hospital transport of critically ill patients. Crit Care 2015; 19:214

84 Gunnarsson T, Theodorsson A, Karlsson P, et al. Mobile computerized tomography scanning in the neurosurgery intensive care unit: increase in patient safety and reduction of staff workload. J Neurosurg 2000;93(03):432-436

85 Peace K, Maloney-Wilensky E, Frangos S, et al. Portable head CT scan and its effect on intracranial pressure, cerebral perfusion pressure, and brain oxygen. J Neurosurg 2011;114(05): $1479-1484$ 